\title{
OPEN SCIENCE UND DIE BIBLIOTHEK - AKTIONSFELDER UND BERUFSBILD
}

\section{von Ina Blümel, Bastian Drees, Christian Hauschke, Lambert Heller und Marco Tullney}

Zusammenfassung: Eine durch die Digitalisierung veränderte und auf Open Science ausgerichtete Wissenschaftspraxis benötigt angepasste Infrastrukturen und Services. Daraus ergeben sich verschiedene neue oder veränderte Aktionsfelder für wissenschaftliche Bibliotheken und Infrastruktureinrichtungen. Zu nennen sind zum Beispiel die nicht-textuellen Materialien wie Forschungsdaten, AV-Medien oder Software und die Umsetzung der FAIR-Prinzipien. Hinzu kommen neue Aufgaben im Bereich der Forschungsinformationen, zum Beispiel in der Unterstützung institutioneller Forschungsinformationssysteme, die Gestaltung von Open Access, die Unterstützung kollaborativen wissenschaftlichen Arbeitens sowie die Schaffung von offenen Infrastrukturen. In diesem Artikel werden diese Felder kurz vorgestellt und sich daraus abzeichnende Anforderungen an das bibliothekarische Berufsbild skizziert.

Schlagwörter: Open Science; Berufsbild; Forschungsinformationen; Open Data; Open Access; wissenschaftliche Bibliotheken

\section{OPEN SCIENCE AND THE LIBRARY - FIELDS OF ACTION AND OCCUPATIONAL PROFILE}

\begin{abstract}
A scientific practice that has been changed by digitalisation and is oriented towards open science requires adapted infrastructures and services. This results in various new or changed fields of action for scientific libraries and infrastructure facilities. Examples include non-textual materials such as research data, audiovisual media or software, and the implementation of FAIR principles. In addition, there are new tasks in the field of research information, for example supporting institutional research information systems, shaping Open Access activities, the support of collaborative scientific work and the creation of open infrastructures. This article briefly presents these fields and outlines the resulting requirements for the library profession.
\end{abstract}

Keywords: Open Science; occupational profile; research information; Open Data; Open Access; academic libraries

DOI: https://doi.org/10.31263/voebm.v72i2.2808 
(c) Ina Blümel, Bastian Drees, Christian Hauschke, Lambert Heller, Marco Tullney

Dieses Werk ist lizenziert unter einer

Creative-Commons-Lizenz Namensnennung 4.0 International

\section{Inhalt}

1. Einführung: Was ist Open Science?

2. Bibliothekarische Open-Science-Aktionsfelder

3. Berufsfeld Open Science in der Bibliothek

4. Fazit

\section{Einführung: Was ist Open Science?}

Eine der Aufgaben wissenschaftlicher Bibliotheken ist die Erbringung von "Dienstleistungen für die Informationsversorgung der Öffentlichkeit bzw. der Institution, der die jeweilige Bibliothek zugeordnet ist" (Gantert 2016, S. 6). Ändert sich die wissenschaftliche Praxis, so ändern sich auch Anforderungen an diese Dienstleistungen. So resultiert die Digitalisierung der Wissenschaft in verschiedene Folgeeffekte und Begleiterscheinungen. Eines der markantesten Charakteristika der sich zunehmend digitaler Dienste und Werkzeuge bedienender Wissenschaft ist die Möglichkeit, Aspekte der Forschungspraxis sowie wissenschaftliche Ergebnisse als Produkt offener, kollaborativer Zusammenarbeit der Öffentlichkeit kostenlos zugänglich zu machen. Ein weiteres Charakteristikum ist die aktive Einbeziehung von Bürger*innen in die Forschung indem Zugang zu Forschungsoutput ermöglicht wird, aber auch im Sinne von Citizen Science. Man spricht in diesem Zusammenhang von Open Science.

Vicente-Saez und Martinez-Fuentes (2018) haben verschiedene Ansätze für Definition und Inhalt von Open Science gesammelt und kommen zu folgender Definition: "Open Science is transparent and accessible knowledge that is shared and developed through collaborative networks" (ebd., S. 434). Gerdes (2018) versucht, eine dichter an das Bibliothekswesen bezogene Auswahl von Open-Science-Definitionen zu treffen. Wegen seiner Fokussierung auf konkrete Aktionsfelder (und deren Motivationen) möchten wir hier einen dieser Ansätze aufgreifen. Fecher und Friesike (2014) unterteilen die Open-Science-Bewegung in fünf Denkschulen (Abb. 1), denen unterschiedliche Motivationen zugrunde liegen: Die Infrastructure School zielt auf die Schaffung von offenen und allen zugänglichen Plattformen, Werkzeugen und Diensten ab, die Public School widmet sich der Öffnung der Wissenschaft für Bürger*innen, die Measurement School entwickelt al- 
ternative Metriken zur Messung wissenschaftlichen Impacts, die Democratic School möchte Wissen für alle zugänglich machen und die Pragmatic School schließlich hat die Effizienzerhöhung wissenschaftlicher Prozesse im Sinn.

Die meisten der unter dem Begriff Open Science einzuordnenden Aktivitäten kann man auf eine oder mehrere dieser Motivationen und somit Denkschulen zurückführen. So ist zum Beispiel die Open-Access-Bewegung, die sich dem freien Zugang und der freien Nachnutzung wissenschaftlicher Publikationen widmet, gleich in mehreren Denkschulen verortbar. Einerseits werden im Namen von Open Access (OA) Infrastrukturen geschaffen, die allen zugänglich sein sollen. OA dient explizit auch der Zugänglichmachung von Wissen, und auch eine erhöhte Effizienz von OA wird oft unterstellt, zum Beispiel im Zusammenhang mit dem Zitationsvorteil von OAPublikationen im Vergleich zu Publikationen hinter einer Paywall (Davis \& Walters 2011; Lewis 2018; Piwowar et al. 2018). ${ }^{1}$ Als weiteres Beispiel können die Initiativen zur Schaffung offener Zitationskorpora dienen, die einerseits der Measurement School, andererseits der Infrastructure School zuzuordnen sind (Klein 2017; Peroni et al. 2017). ${ }^{2}$

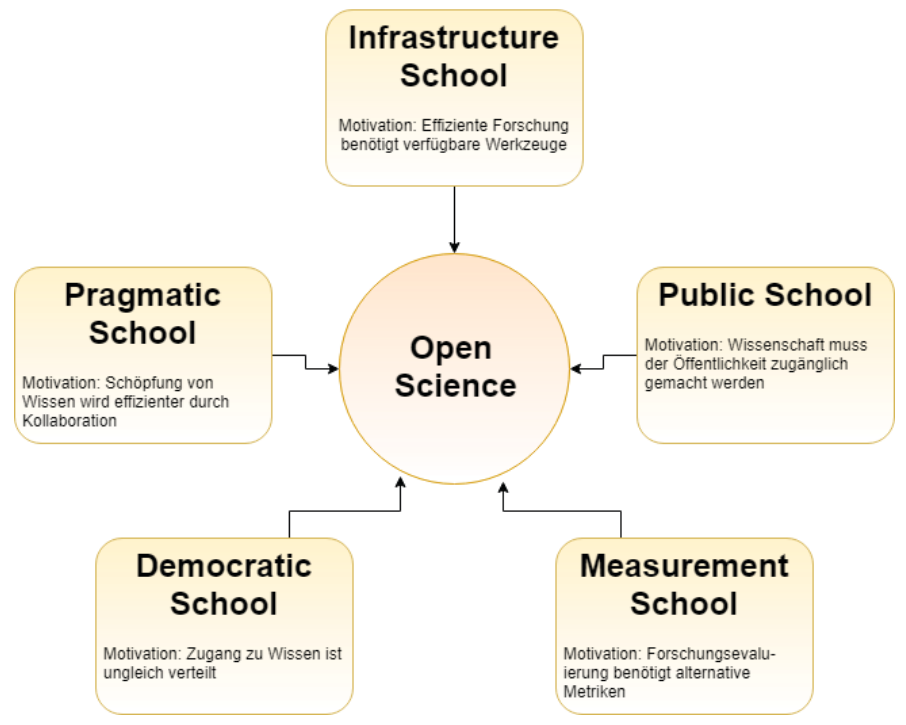

Abb. 1: Nach den Five Schools of Open Science (Fecher und Friesike 2014, S. 19)

Die Entwicklung der Wissenschaft in Richtung Open Science wird von verschiedenen Akteur*innen getrieben. ${ }^{3}$ Da sind einerseits aus der wissenschaftlichen Community selbst vorangetriebene Aktivitäten, andererseits 
werden diese Ideen inzwischen vermehrt von staatlichen und nichtstaatlichen Interessensgruppen aufgegriffen. Genannt sei hier zum Beispiel die weitreichende Initiative vieler (öffentlicher und privater) Förderorganisationen zur beschleunigten Umstellung auf Open Access Plan $\mathrm{S}^{4}$ oder die von der Politik- und Unternehmensberatungsfirma PriceWaterhouseCoopers (PwC EU Services 2018) durchgeführte Studie zu den wirtschaftlichen Effekten von FAIR Research Data ${ }^{5}$ (siehe Abb. 1).

\section{Bibliothekarische Open-Science-Aktionsfelder}

Es ist im Rahmen dieses Artikels nicht möglich, eine umfassende Übersicht aller aktuellen Open-Science-Aktivitäten und Trends zu schaffen, die Auswirkungen auf Bibliotheken, ihre Mitarbeiter*innen sowie deren Aufgabenfelder haben. In Anlehnung an die Fachliteratur (vgl. Gerdes 2018; Ogungbeni et al. 2018; Schmidt et al. 2018; Senst \& Heldt 2017) und die von uns in unserem professionellen Umfeld wahrgenommenen Aktivitäten möchten wir eine Auswahl an relevant erscheinenden Entwicklungen vorstellen und versuchen, diese in das Spektrum der fünf Open-ScienceDenkschulen einzuordnen. Hierbei soll der Fokus vor allem auf der Rolle der Bibliotheken liegen.

\subsection{Open-Access-Publizieren unterstützen}

Open Access ist schon länger als andere Open-Science-Komponenten ein Thema in wissenschaftlichen Bibliotheken. Eine quantitative Ausweitung (zusätzliche Tätigkeiten, steigende Zahl von Open-Access-Veröffentlichungen) und eine qualitative Veränderung der Instrumente (Einbindung in den Publikationsprozess) verändern jedoch die Anforderungen an Bibliotheken. Vielfach tauchen neue Tätigkeitsfelder auf, die nur bedingt an klassische Tätigkeiten anknüpfen können. Die Rolle, die Bibliotheken dabei einnehmen, muss definiert werden; dies betrifft nicht nur eine Klärung der eigenen Funktion, sondern auch eine Auseinandersetzung mit der Außenwahrnehmung, z.B. durch Wissenschaftler*innen und Wissenschaftspolitik: Wird die Bibliothek überhaupt als zuständig oder kompetent für Fragen, die sich aus dem Forschungsprozess oder aus dem Dialog mit Forschungsförderern ergeben, eingestuft?

Besonders deutlich zeigt sich ein solcher Wechsel der Aufgaben und Anforderungen dort, wo Bibliotheken aktiv am Publikationsprozess beteiligt sind, z.B. durch den Betrieb von Universitätsverlagen, Zeitschrif- 
tenservern und anderen Publikationsplattformen. Hier übernehmen sie Verantwortung für Qualitätssicherung, redaktionelle Aufgaben, Sicherheit, Archivierung etc. in einem anderen Maß als bisher (bei der retrospektiven Erfassung, Sammlung, Zugänglichmachung und Archivierung bereits andernorts veröffentlichter Werke), auf der anderen Seite werden andere Bedürfnisse berücksichtigt und Kompromisse geschlossen. Die Unterstützung von Wissenschaftler*innen beim Publizieren ist eine Nebenrolle, in der Bibliotheken beraten können, aber in aller Regel nicht entscheiden (und Verantwortung übernehmen) können, wo und wie publiziert wird.

Zu den relevanten Aufgaben gehören:

- Beratung von Wissenschaftler*innen und Bibliothekar*innen in einem komplexen Feld stets unterschiedlicher Open-Access-Konditionen und -Auflagen inklusive der Minderung von Rechtsunsicherheit bei den Ratsuchenden

- Betrieb von Repositorien, die Unterstützung von Autor*innen und die Übernahme von Restrisiken (z.B. bei Zweitveröffentlichungen)

- Betrieb von Publikationsplattformen für Zeitschriften, Bücher etc. Hier ist redaktionelle und verlagsartige Arbeit nötig, und es müssen (über Test- und Pilotfälle hinaus) Skalierungsoptionen mitgedacht werden. Auch die Orientierung auf Qualität und die Vermeidung eines Hausverlag-Eindrucks sind wichtig.

- Betrieb von Publikationsfonds, inklusive ihrer Weiterentwicklung (vgl. Pampel/Tullney 2017)

- Konzeption und Unterstützung von Open-Access-Finanzierungsmodellen, die besser skalieren und nachhaltiger sind als das vorherrschende APC-Modell. Weiterentwicklung von Erwerbungsbudgets, Finanzierung von Pilotvorhaben (Beispiel: Unterstützung des Journal Flippings des Journal of Informetrics zu Quantitative Science Studies ${ }^{6}$ ) und Beteiligung an entsprechenden Open-Access-Konsortien.

- Vermeidung von double dipping (Mehrfachfinanzierung von Publikationen aus Subskriptions- und Open-Access-Mitteln): Finanzierung von Open Access auf der Basis vorhandener Strukturen, Mittel und Zuständigkeiten in der Bibliothek, keine Etablierung dauerhafter Mehrfachfinanzierung

- Monitoring von Open-Access-Anteilen und Open-Access-Ausgaben (vgl. Jahn/Tullney 2016)

- Weiterentwicklung von Open-Access-Modellen in Richtung bisher unterrepräsentierter Disziplinen (nicht nur im geisteswissenschaftlichen Bereich) und Publikationstypen (z.B. Bücher, Konferenzpubli- 
kationen), auch auf Basis internationaler Zusammenarbeit; Unterrepräsentation zeigt sich z.B. in Open-Access-Anteilen, bereitstehenden Open-Access-Optionen etc.

- Initiierung und Unterstützung von Policy-Vorhaben auf institutioneller, regionaler, nationaler und internationaler Ebene: Open-Access- und Open-Science-Policies, Handreichungen, Beobachtung der Wirksamkeit (aktuell insbesondere Plan S)

Die Rolle der Bibliothek im Umfeld des wissenschaftlichen Publizierens ändert sich, und die Bibliothek muss diese Rolle selbst definieren. Da nicht nur für Sachmittel, sondern auch für Personalmittel gilt, dass es zu einer Umstellung und nicht zu einer immer weiteren Ausdehnung kommen wird, muss darüber gesprochen werden, wie neue Aufgaben möglichst gut in das bisherige Tätigkeitsspektrum eingefügt werden können und welche Aufgaben zukünftig weniger nachgefragt werden. Gleichzeitig ist es zentral, die Grenzen der eigenen Kompetenzen und Aufgaben zu kennen und neue Tätigkeiten sinnvoll auszuwählen. Im Open-Access-Bereich ist man zudem mit massiven finanziellen Interessen großer kommerzieller Akteure konfrontiert (darunter große traditionelle Wissenschaftsverlage wie Elsevier, aber auch reine Open-Access-Verlage), was widerständiges und selbstbewusstes Agieren notwendig macht. Dies hilft nicht nur der eigenständigen Rolle der Bibliothek, sondern trägt auch dazu bei, nachhaltige und finanzierbare Open-Access-Modelle zu etablieren.

Wie einleitend beschrieben, lassen sich die Open-Access-Aktivitäten, je nach Schwerpunkt, unterschiedlichen Denkschulen zuordnen. Vorwiegend kann die Open-Access-Bewegung jedoch der Democratic School zugeordnet werden, deren zentrales Ziel es ist, wissenschaftliche Informationen für jeden frei zugänglich zu machen.

\subsection{AV-Medien, Forschungsdaten, Software: Open Data unterstützen}

Eng verwandt mit Open-Access-Aktivitäten sind alle Bestrebungen jeglichen wissenschaftlichen Output, jenseits klassischer Textpublikationen, frei zugänglich und nachnutzbar zu machen. Dies sind auf der einen Seite die den Forschungsergebnissen zugrunde liegenden Daten sowie die zu deren Erzeugung oder Verarbeitung verwendete Software, auf der anderen Seite aber auch das Verständnis ermöglichende oder Daten interpretierende Visualisierungen wie Grafiken, 3D-Modelle, Animationen, Simulationen oder Videos. Zielen die Aktivitäten in diesem Bereich eher auf eine Ergänzung von Open Access im Sinne des freien Zugangs zu wissenschaftlichen 
Ergebnissen ab, so lassen sie sich ebenfalls der Democratic School zuordnen. Andererseits stehen bei Open Data und Open Source häufig die Nachnutzbarkeit und damit die Vermeidung von Doppelarbeit im wissenschaftlichen Betrieb im Vordergrund. In diesem Sinne können die Aktivitäten in erster Linie der Pragmatic School zugeordnet werden.

Für wissenschaftliche Bibliotheken sind viele dieser Aktionsfelder, wie z.B. Forschungsdatenmanagement, keineswegs neu, sondern gehören bereits seit längerem zum Standardrepertoire der meisten Infrastruktureinrichtungen. Dennoch gibt es hier großen Verbesserungsbedarf u.a. hinsichtlich der Nachnutzbarkeit sowie Maschinen- und Menschenlesbarkeit von Forschungsdaten. Hier spielen Metadatenstandards für Forschungsdaten (DataCite 2019) und Zitationsprinzipien für Software (Smith 2016) eine zentrale Rolle, um Reproduzierbarkeit und Nachnutzbarkeit der Ergebnisse für die Wissenschaft zu gewährleisten. Ferner kommen der Interoperabilität und Maschinenlesbarkeit der (Meta-)Daten eine große Bedeutung zu, insbesondere für automatisierte Analysen und Methoden wie Text und Data Mining. Gleichzeitig sind vergleichbare Aktivitäten im Bereich wissenschaftlicher Software, d.h. ein Management von Forschungssoftware, in Bibliotheken bisher kaum etabliert. Darüber hinaus sind für andere nicht-textuelle Materialien, wie audiovisuelle Medien oder 3D-Objekte, medienspezifische Lösungen erforderlich (Strobel 2014), welche nur sehr selten von Bibliotheken angeboten werden.

Den Infrastruktureinrichtungen kommt die zentrale Aufgabe zu, „Mensch und Maschine bei der Auffindung, dem Zugang, der Integration und der Analyse von wissenschaftlichen Daten zu unterstützen". Als Leitlinie können die FAIR Data Principles verstanden werden, welche 2016 von einer Arbeitsgruppe im Rahmen von FORCE11 ${ }^{8}$ veröffentlicht wurden (Wilkinson 2016). Bei diesen Prinzipien steht FAIR für Findable (auffindbar), Accessible (zugänglich), Interoperable (interoperabel) und Reusable (nachnutzbar). Auch wenn die FAIR-Prinzipien durch Open Science inspiriert wurden, sind diese beiden Bewegungen nicht gleichzusetzen (Mons 2017). Allerdings erfordern die FAIR-Prinzipien "Klarheit und Transparenz über die Bedingungen für den Zugang und die Wiederverwendung" der Daten, auch wenn diese nicht "open" sind".

Die Rolle der Bibliotheken muss es dabei sein, einerseits Infrastrukturen - wie z.B. Forschungsdatenrepositorien - zur Verfügung zu stellen, die sich an den FAIR-Prinzipien orientieren, und zu versuchen ihre 'FAIRness' kontinuierlich zu steigern, d.h. eine größtmögliche Übereinstimmung mit den FAIR-Prinzipien zu erreichen. Um diese,FAIRness' möglichst objektiv und der jeweiligen Fachcommunity angemessen bestimmen 
und messen zu können, wurde von der FAIR Metrics group ${ }^{10}$ ein entsprechender Rahmen zur Selbstevaluierung der ,FAIRness' geschaffen (Wilkinson 2018). Andererseits spielt die Bibliothek die Rolle des Bindeglieds zwischen Datenproduzent*innen und Nachnutzenden und kann beide mit ihrer Expertise, vor allem in den Bereichen Metadaten und Lizenzen, unterstützen.

\subsection{Forschungsinformationen extrahieren, strukturieren und öffnen}

Forschungsinformationen erfahren erhöhte Aufmerksamkeit einerseits durch verstärkte Berichtsanforderungen von Forschungsförderern und Trägern wissenschaftlicher Einrichtungen, andererseits durch stetig steigende Transparenzanforderungen der Öffentlichkeit an die Wissenschaft. Mit Forschungsinformationen sind Informationen über „Projekte, Publikationen, publizierte Datensätze, Infrastrukturen und Personen bzw. Teams“ (Ebert et al. 2016, S. 7) gemeint. Sowohl die Pflege der Daten als auch die Definition und Anpassung der zugrunde liegenden Datenstandards und Ontologien ist eine im Kern bibliothekarische Angelegenheit. Insbesondere für größere Bibliotheken kann auch der Betrieb von Forschungsinformationssystemen sowie Import und Validierung von Metadaten eine neue und geeignete Aufgabe sein (vgl. Einbock \& Hauschke 2018; Thiedig 2018).

Darüber hinaus muss es Betätigungsfeld des Bibliothekswesens sein, gemeinsam eine Infrastruktur für und einen Korpus von frei zugänglichen Forschungsinformationen mit einer hohen Aktualität und Qualität in Einklang mit der School of Measurement zu schaffen. ${ }^{11}$ Dies könnte die technische Bereitstellung eines Wissensgraphen mit Informationen über wissenschaftliche Akteure, ihre Verbindungen und ihren Output, und auch die inhaltliche Arbeit an diesem Graphen. Tullney (2017) stellt fest, dass freie und vollständige Bibliografien inklusive Informationen über den Open-Access-Status erforderlich seien, um Open-Access-Monitoring durchführen zu können, und die San Francisco Declaration on Research Assessment (DORA) fordert transparente und nachnutzbare Daten für die Forschungsevaluierung (Cagan, R. 2013). Der reichhaltige bibliothekarische Erfahrungsschatz in der kollaborativen Erstellung von Katalogen und Bibliographien kann angewendet werden, um gemeinsam an solch einer offenen Datenbasis für Forschungsinformationen und wissenschaftsevaluierenden (alternativen) Metriken, wie sie u.a. in der - vielleicht im Sinne von FAIR Research Information analog zu FAIR Research Data - zu arbeiten. 


\subsection{Fachcommunities durch offene Formen der Zusammenarbeit unterstützen}

Forschungsdaten werden heute zumeist in digitaler Form erhoben und analysiert. An der Auswertung kann von den Co-Autor*innen online gemeinsam gearbeitet und das Ergebnis gemeinsam geschrieben werden. Dieser Aspekt der kollaborativen wissenschaftlichen Arbeit steht im Zentrum der Pragmatic School. Ein gewichtiger Aspekt im Kontext dieser umfassenden Digitalisierung wissenschaftlicher Tätigkeiten und Prozesse sind die mittelbaren Effekte auf die Arbeitsweisen auf der sozialen Ebene. So entstand in der Community um freie Software und freie Infrastruktur die Idee, "Sprints" (bis dahin bekannt als soziale Methode in der agilen Softwareentwicklung) auf das Schreiben von Büchern zu übertragen - mit dem Ergebnis der Book Sprints, einer heute weltweit etablierten Methode zur effektiven gemeinschaftlichen Entwicklung von Dokumentationen, Lehrund Handbüchern (Schrenk und Mehlberg 2015).

Book Sprints sind dabei nur ein Beispiel für die Interaktion mit Fachcommunities, die von neuen, digital inspirierten Arbeitsweisen profitieren können. Ein anderes Beispiel wären etwa die Hands-On-Labs, die 2017 als neuer Veranstaltungstyp beim Deutschen Bibliothekartag eingeführt wurde, der jährlich stattfindenden, größten bibliothekarischen Bildungsveranstaltung Europas. Die Hands-On-Labs dienen den bibliothekarischen Berufsverbänden als handlungsorientiertes Format, dass auf die sich verändernden Bedarfe von Fachbesucher*innen zugeschnitten ist (Heller 2017). In ihnen kann beispielsweise die Installation oder der Einsatz einer Software erprobt werden.

Ein weiteres Beispiel sind die Open-Science-Barcamps, die seit 2015 jährlich vom Leibniz-Forschungsverbund Open Science ausgerichtet werden. Auch hier wird der Wissensaustausch von Fachleuten durch ein Format unterstützt, bei dem nicht vorgegebene Rollen (zum Beispiel Vortragende und Teilnehmende, oder Lehrende und Lernende) und vordefinierte Ergebnisse im Mittelpunkt stehen, sondern das Wissen und Interesse von den Teilnehmenden in eigener Verantwortung eingebracht, verbunden und erweitert wird. Die internationale Teilnehmer*innenschaft von digital interessierten Wissenschaftler*innen sowie Mitarbeiter*innen von Infrastruktureinrichtungen und Unternehmen ist inzwischen so stark angewachsen, dass eine häufigere Frequenz dieser Barcamps als sinnvoll erscheint.

Die genannten Beispiele zeigen, wie

- sich die berufliche Weiterbildung von Bibliothekar*innen weiterentwickeln lässt - angesichts sich dynamisch entwickelnder Anforde- 
rungen an das berufliche Wissen und Können (siehe Abschnitt „Berufsfeld Open Science in der Bibliothek")

- neue Medien- und Dienste-Typen entwickelt und vermarktet werden können, indem die potentiellen Zielgruppen nicht nur passiv adressiert, sondern in gemeinsames Handeln einbezogen werden - bis hin zu der Option, gemeinsam Drittmittel für das Bibliothekswesen oder das Wissenschaftssystem relevante Forschung und Entwicklung einzuwerben.

\subsection{Offene und nachnutzbare Infrastrukturen schaffen und erhalten}

Die Entwicklung des wissenschaftlichen Sektors hin zu mehr Offenheit ist insbesondere hinsichtlich wissenschaftlicher Infrastruktur gemäß der School of Infrastructure generell positiv zu betrachten. Die in Projekten einer Einrichtung geschaffene Open-Source-Software kann von anderen Einrichtungen nachgenutzt werden, die Deutungshoheit und Verfügungsgewalt über die geschaffene Infrastruktur kann bei der wissenschaftlichen Community verbleiben (Schlosser 2019). Die für die Entwicklung der Software eingesetzten Mittel erzeugen einen Wert über die eigene Einrichtung hinaus. ${ }^{12}$

Dies setzt allerdings voraus, dass die Software-Entwicklung nachhaltig durchgeführt wird. Dazu gehört, wie oben angeführt, die Langzeitarchivierung der Software, doch auch die aktive Mitarbeit in Open-Source-Projekten an der Software selbst kann bibliothekarische Aufgabe sein, insbesondere da im wissenschaftlichen Sektor in vielen Bereichen Open-SourceLösungen eingesetzt werden wie zum Beispiel Open Journal Systems (Willinsky 2005) für das Hosting von Zeitschriften, die Repository-Software DSpace (Smith et al. 2003), das Forschungsinformationssystem VIVO (Conlon et al. 2019) oder auch das integrierte Bibliothekssystem Koha (vgl. Villányi 2017). Beiträge zur Weiterentwicklung dieser Software - ob direkte Mitarbeit in der Software-Entwicklung, der Übersetzung, Mitarbeit in Beiräten und anderen Gremien oder die finanzielle Beteiligung durch Mitgliedschaft in Trägervereinen - sind notwendig, um ihr Nachhaltigkeit zu verschaffen.

Über die Arbeit an Open-Source-Software hinaus ist auch die Schaffung von Plattformen und technischen Diensten eine wichtige Aufgabe für Bibliotheken im Open-Science-Sektor, wie z.B. die offene Bereitstellung von Vokabularen in menschen- und maschinenlesbarer Form oder die Mitarbeit an Initiativen wie der oben bereits erwähnten Initiative for Open Citations. Ein weiteres Beispiel ist die Gewährleistung der dauerhaften 
Verfügbarkeit und Nachnutzbarkeit wissenschaftlicher Daten und Software, um einerseits die Reproduzierbarkeit wissenschaftlicher Ergebnisse zu gewährleisten und andererseits zeit- und kostenintensive Mehrarbeit zu vermeiden, die entsteht, wenn Daten und Software von jedem/jeder Wissenschaftler*in neu produziert werden müssen. Hier spielen Infrastruktureinrichtungen wie Bibliotheken und Rechenzentren eine zentrale Rolle, indem sie Dienste zur Publikation und Archivierung von Forschungsdaten und -software sowie anderer Materialien wie audiovisueller Medien bereitstellen (Kraft et al. 2017) - insbesondere, wenn sie in der Hand und unter der Kontrolle der wissenschaftlichen Community verbleiben sollen. Über den Betrieb eigener Infrastrukturen hinaus spielen Bibliotheken eine wichtige Rolle bei der gemeinschaftlichen Finanzierung offener Infrastrukturen. $^{13}$

Ein dritter Aspekt der offenen Infrastrukturen ist die Arbeit an und die Bereitstellung von wissenschaftsunterstützenden Daten wie zum Beispiel die Extraktion von Zitationsinformationen aus Monographien (Lauscher et al. 2018) oder auch bei der Entitätenerkennung (Bargheer \& Bertino 2018).

\subsection{Den Open-Science-Diskurs führen und begleiten}

Neben den bisher genannten Aktionsfeldern ist der Diskurs über Open Science und die Aufklärung zu entsprechenden Handlungspraktiken eine Aufgabe, die Bibliotheken frei Haus geliefert wird. Hier gilt es, Synergieeffekte zu nutzen: Wenn Forschende das Gespräch über Open-Access-Auflagen suchen, bietet es sich an, sie mit Dienstleistungen, Angeboten oder aktuellen Entwicklungen im Open-Science-Kontext vertraut zu machen. Diese Konversationen über Open Science erfüllen verschiedene Funktionen: Akzeptanzprobleme können offensiv angegangen werden sowie Unterstützungsmöglichkeiten für die Forschenden identifiziert werden, und die Bibliothekar*innen etablieren sich als Teil der wissenschaftlichen Community of Practice (Heller 2008).

Darüber hinaus ist es durchaus angebracht, aktiv für Open-SciencePrinzipien zu werben. Dazu gehört nicht nur die Werbung für Open-Science-Praktiken bei den Forschenden, sondern auch die Unterstützung von Initiativen wie DORA zur fairen und transparenten Forschungsevaluierung. Wenn Bibliotheken sich der wichtigen Aufgabe widmen, Infrastruktur selbstständig oder in Kooperation mit anderen zu betreiben, ist es ebenfalls wichtig, Open-Science-Kriterien anzuwenden und selber zu praktizieren. 


\section{Berufsfeld Open Science in der Bibliothek}

Im Kontext von Open Data und Open Science wachsen neue Tätigkeitsfelder, während sich bisherige verändern. Hapke (2016) stellt die Veränderungen im Berufsbild der Bibliothekar*innen in Praxis und Studium sowie die Abgrenzung neuer Berufsbezeichnungen unter dem Titel "Data Librarian" vor. Diese neuen beruflichen Arbeitsfelder in Bibliotheken und Informationseinrichtungen sind oftmals an der Schnittstelle zu Wissenschaft und/oder Rechenzentren angesiedelt. Begleitet wird die Entwicklung von aktuellen Projekten auf nationaler, regionaler und europäischer Ebene, wie z.B. dem Projekt der European Open Science Cloud (EOSC) ${ }^{14}$, das versucht, eine offene Plattform und Infrastruktur für den Austausch von FAIR Data zu schaffen. Die High Level Expert Group on the European Open Science Cloud ${ }^{15}$ schätzte 2016, dass eine halbe Million „core data scientists“ benötigt würden und empfahl die Finanzierung von "gezielten Maßnahmen zur Entwicklung von Kernkompetenzen im Datenbereich”. Der deutsche Rat für Informationsinfrastrukturen $(R f l I)^{16}$ argumentiert in die gleiche Richtung und empfahl im selben Jahr die Förderung der allgemeinen und fachlichen Kompetenzentwicklung des wissenschaftlichen Nachwuchses in Bezug auf den Bereich. Neue Berufsbilder (wie Datenarchivar*in, digitaler Dokumentar*in, Datenbibliothekar*in, Data Scientist, Datenkurator*in) sollten durch entsprechende Vollstudiengänge ermöglicht werden, um die Lücke zwischen Wissenschaftler*innen und Informationsinfrastrukturen zu schließen (vgl. Petras et al. 2019). Daher ist es notwendig, das aktuelle Berufsbild auch (und nicht nur) auf neue Anforderungen aus der Open Science zu prüfen und anzupassen sowie entsprechende Folgen für die Ausund Weiterbildung zu ermitteln und umzusetzen. Erste Schritte zu einer Auswertung der aktuell gefragten Kompetenzen wurden bereits unternommen (Zellmann 2018), ein automatisiertes Monitoring scheint darüber hinaus auch als erstrebenswert (Blümel et al. 2019).

Es ist jedoch ebenso wichtig, nicht nur zukünftiges Personal in Berufsausbildung und Studium auf berufliche Herausforderungen vorzubereiten. Der Einbeziehung und Qualifizierung vorhandenen Personals kommt eine äußerst wichtige Rolle zu. Antworten auf diese Herausforderung muss die bibliothekarische Weiterbildung finden. Denn obwohl nicht jede Prophezeiung der Vergangenheit hinsichtlich neuer Aktionsfelder sofort oder überhaupt bibliothekarischer Alltag wurde/wird, ist eine Beschäftigung mit Trends und Entwicklungen in der wissenschaftlichen Praxis generell zwingend erforderlich. Dazu kommt die Sensibilisierung des bibliothekarischen Personals für kritische Aspekte von Open Science, wie zum Beispiel 
ethisch heikle Anwendungen von Big Data, Datenschutzprobleme beim User Tracking oder die Missbrauchspotentiale von publizierten oder neu verknüpften Daten. Im Einzelfall kann es erforderlich sein, Daten - zum Beispiel über Populationen geschützter Arten - nicht oder nur bearbeitet zu publizieren (Tulloch et al. 2018).

\section{Fazit}

Neben den in diesem Artikel angerissenen Aktionsfeldern und veränderten Anforderungen durch neue wissenschaftliche Handlungspraktiken, gibt es zahlreiche weitere für Bibliotheken relevante Aktionsfelder im Open-Science-Kontext. Hier sind u.a. die Felder der sogenannten Citizen Science (vgl. Public School) sowie der Open Educational Resources zu nennen. Die Aufgaben der Bibliotheken können hier u.a. das Bereitstellen von OER, die Beratung zu Herstellung und Produktion von OER, Schulungen zum Umgang mit OER und die Verbreitung von OER sein (Fahrenkrog 2017, Stummeyer 2018).

Die Rolle der Bibliothek im Open-Science-Kontext muss für jedes Umfeld spezifisch beantwortet werden. Vorbild für eine derartige Analyse kann die Bibliothek des Robert-Koch-Instituts sein, die den Forschungszyklus ihrer Institution aufschlüsselte und spezifizierte, welche Dienstleistungen die Bibliothek an welcher Stelle erbringen kann. Dort wurde der Fokus von der Frage "Was können wir anbieten?” auf „Was brauchen die Wissenschaftler*innen?" verschoben (Senst \& Heldt 2017). Open Science lässt es in vielen Fällen zu, diese Frage so zu formulieren, dass beide Fragen gleich mit beantwortet werden: Was braucht die Wissenschaft? Grundsätzlich wandelt sich die Rolle der Bibliothek: von der Verwaltung von Sammlungen und einer klar umrissenen, zentralen Aufgabe hin zu einer kollaborativen Akteurin in einer dezentralen, offenen Forschungslandschaft (vgl. Revez 2018). Dies bedingt eine neue Identität der Bibliothek und angepasste Wahrnehmungen des Berufsbilds.

Obwohl es richtig und wichtig ist, Entwicklungen zu beobachten und auf sie zu reagieren, ist es darüber hinaus genauso sinnvoll, selbst aktiv zu werden. Bibliotheken sollten den Mut haben, nicht nur ausgetretenen Pfaden zu folgen oder zu warten, bis sich das Feld sortiert hat, sondern selbst neue Wege beschreiten. Dabei ist Austausch und Zusammenarbeit zwischen Wissenschaft und Bibliothekswesen, aber auch innerhalb und unter den Bibliotheken zwingend erforderlich, um Doppelarbeit und Insellösungen zu vermeiden und Herausforderungen wirksam begegnen zu 
können. Das Ziel, eine von der Community geführte und gesteuerte Wissenschaft zu gestalten, kann nur gemeinsam erreicht werden.

Dr. in Ina Blümel

ORCID: https://orcid.org/0000-0002-3075-7640

Technische Informationsbibliothek (TIB)

E-Mail: ina.bluemel@tib.eu

Dr. Bastian Drees

ORCID: https://orcid.org/0000-0003-3508-602X

Technische Informationsbibliothek (TIB)

E-Mail: bastian.drees@tib.eu

Christian Hauschke

ORCID: https://orcid.org/0000-0003-2499-7741

Technische Informationsbibliothek (TIB)

E-Mail: christian.hauschke@tib.eu

Lambert Heller

ORCID: https://orcid.org/0000-0003-0232-7085

Technische Informationsbibliothek (TIB)

E-Mail: lambert.heller@tib.eu

Marco Tullney

ORCID: https://orcid.org/0000-0002-5111-2788

Technische Informationsbibliothek (TIB)

E-Mail: marco.tullney@tib.eu 


\section{Literatur}

Bargheer, Margo; Bertino, Andrea C. (2018): HIRMEOS. In: Bibliothek Forschung und Praxis 42 (3), S. 476-485. https://doi.org/10.1515/bfp2018-0056

Blümel, Ina; Heller, Lambert; Hermes, Jürgen; Tu, Pascal Ngoc Phu; Witt, Andreas; Zellmann, Cedrik (2019): Kompetenzmonitor OpenBiblioJobs (Zenodo). https://doi.org/10.5281/zenodo.2561309

Cagan, Ross (2013): The San Francisco Declaration on Research Assessment. In: Disease models \& mechanisms 6 (4), S. 869-870. https://doi. org/10.1242/dmm.012955

Conlon, Michael; Woods, Andrew; Triggs, Graham; O'Flinn, Ralph; Javed, Muhammad; Blake, Jim et al. (2019): VIVO: a system for research discovery. In: Journal of Open Source Software 4 (39), 1182. https://doi. org/10.21105/joss.01182

DataCite Metadata Working Group (2019): DataCite Metadata Schema Documentation for the Publication and Citation of Research Data v4.2. Unter Mitarbeit von Madeleine de Smaele, Amy Hatfield Hart, Jan Ashton, Isabel Bernal Martínez, Stefanie Dietiker, Jannean Elliott et al. https://doi.org/10.5438/bmjt-bx77

Davis, Philip M.; Walters, William H. (2011): The impact of free access to the scientific literature: a review of recent research. In: Journal of the Medical Library Association: JMLA 99 (3), S. 208-217. https://doi. org/10.3163/1536-5050.99.3.008

Ebert, Barbara; Tobias, Regine; Beucke, Daniel; Bliemeister, Andreas; Friedrichsen, Eiken; Heller, Lambert et al. (2015): Forschungsinformationssysteme in Hochschulen und Forschungseinrichtungen. Positionspapier. Version 1.1. https://doi.org/10.5281/zenodo.14828

Einbock, Joanna; Hauschke, Christian (2018): Anforderungen an Forschungsinformationssysteme in Deutschland durch Forschende und Forschungsadministration - Zusammenfassung zweier Studien. In: Informationspraxis 4 (1). https://doi.org/10.11588/ip.2018.1.46819

Fahrenkrog, Gabriele; Plieninger, Jürgen (2017): Management von Open EducationalResources (OER) in Bibliotheken. In: Cornelia Vonhof und Konrad Umlauf(Hg.): Erfolgreiches Management von Bibliotheken und Informationseinrichtungen. 59. Aktualisierungslieferung. Hamburg: Dashöfer. https://biboer.files.wordpress.com/2018/03/oer_in_bibliotheken_embi_17318.pdf

Fecher, Benedikt; Friesike, Sascha (2014): Open Science: One Term, Five Schools of Thought. In: Sönke Bartling und Sascha Friesike (Hg.): 
Opening Science. Cham: Springer International Publishing, S. 17-47. https://doi.org/10.1007/978-3-319-00026-8_2

Gantert, Klaus (2016): Bibliothekarisches Grundwissen. 9., vollständig aktualisierte und erweiterte Auflage. Berlin, Boston: DE GRUYTER SAUR. Gerdes, Thomas (2018): Die Open-Science-Bewegung und ihre Bedeutung für die wissenschaftlichen Bibliotheken (Berliner Handreichungen zur Bibliotheks- und Informationswissenschaft, 428). https://doi. org/10.18452/18983

Heller, Lambert (2008): Bibliothek 2.0 - Perspektiven, Probleme, Handlungsbereiche. In: Christoph Hobohm und Konrad Umlauf (Hg.): Erfolgreiches Management von Bibliotheken. Hamburg: Dashöfer.

Heller, Lambert (2015): Neue Aufgaben für wissenschaftliche Bibliotheken: Das Beispiel Open Science Lab. In: o-bib. Das offene Bibliotheksjournal 2 (3), S. 29-36. https://doi.org/10.5282/o-bib/2015H3S29-36

Heller, Lambert (2017): Hands-on Labs - oder: Dinge gemeinsam herausfinden und ausprobieren beim Bibliothekartag! In: BuB - Forum Bibliothek und Information 69 (5), S. 242. https://nbn-resolving.org/ urn:nbn:de:0290-opus4-141206

High Level Expert Group on the European Open Science Cloud (2016): Realising the European open science cloud. First report and recommendations of the Commission high level expert group on the European open science cloud. Luxembourg. https://doi.org/10.2777/940154 Jahn, Najko; Tullney, Marco (2016): A study of institutional spending on open access publication fees in Germany. In: PeerJ 4, e2323. https:// doi.org/10.7717/peerj.2323

Klein, Annette (2017): Von der Schneeflocke zur Lawine: Möglichkeiten der Nutzung freier Zitationsdaten in Bibliotheken. In: o-bib. Das offene Bibliotheksjournal 4 (4), S. 127-136. https://doi.org/10.5282/obib/2017H4S127-136

Kraft, Angelina; Dreyer, Britta; Löwe, Peter; Ziedorn, Frauke (2017): 14 Years of PID Services at the German National Library of Science and Technology (TIB): Connected Frameworks, Research Data and Lessons Learned from a National Research Library Perspective. In: Data Science Journal 16 (1/2), S. 233. https://doi.org/10.5334/dsj-2017-036

Lauscher, Anne; Eckert, Kai; Galke, Lukas; Scherp, Ansgar; Rizvi, Syed Tahseen Raza; Ahmed, Sheraz et al. (2018): Linked Open Citation Database. In: Jiangping Chen, Marcos André Gonçalves, Jeff M. Allen, Edward A. Fox, Min-Yen Kan und Vivien Petras ( $\mathrm{Hg}$.): Proceedings of the $18^{\text {th }}$ ACM/IEEE on Joint Conference on Digital Libraries - JCDL '18. the $18^{\text {th }}$ ACM/IEEE. Fort Worth, Texas, USA, 03.06.2018-07.06.2018. 
New York, New York, USA: ACM Press, S. 109-118. https://doi. org/10.1145/3197026.3197050

Lewis, Colby Lil (2018): The Open Access Citation Advantage: Does It Exist and What Does It Mean for Libraries? In: ITAL 37 (3), S. 50. https:// doi.org/10.6017/ital.v37i3.10604

Lewis, David W.; Goetsch, Lori; Graves, Diane; Roy, Mike (2018): Funding community controlled open infrastructure for scholarly communication: The $2.5 \%$ commitment initiative. In: $c r l n 79$ (3), S. 133. https:// doi.org/10.5860/crln.79.3.133

Mons, Barend; Neylon, Cameron; Velterop, Jan; Dumontier, Michel; da Silva Santos, Luiz Olavo Bonino; Wilkinson, Mark D. (2017): Cloudy, increasingly FAIR; revisiting the FAIR Data guiding principles for the European Open Science Cloud. In: ISU 37 (1), S. 49-56. https://doi. org/10.3233/ISU-170824

Ogungbeni, John I.; Obiamalu, Amaka R.; Ssemambo, Samuel; Bazibu, Charles M. (2018): The roles of academic libraries in propagating open science. In: Information Development 34 (2), S. 113-121. https://doi. org/10.1177/0266666916678444

Pampel, Heinz; Tullney, Marco (2017): 3b. Open-Access-Publikationsfonds. In: Konstanze Söllner und Bernhard Mittermaier (Hg.): Praxishandbuch Open Access. Berlin, Boston: De Gruyter, S. 162-172. https://doi.org/10.1515/9783110494068-019

Peroni, Silvio; Shotton, David; Vitali, Fabio (2017): One Year of the OpenCitations Corpus. In: Claudia d'Amato, Miriam Fernandez, Valentina Tamma, Freddy Lecue, Philippe Cudré-Mauroux, Juan Sequeda et al. (Hg.): The Semantic Web - ISWC 2017, Bd. 10588. Cham: Springer International Publishing (Lecture Notes in Computer Science), S. 184192. https://doi.org/10.1007/978-3-319-68204-4_19

Petras, Vivien; Kindling, Maxi; Neuroth, Heike; Rothfritz, Laura (2019): Digitales Datenmanagement als Berufsfeld im Kontext der Data Literacy. In: ABI Technik 39 (1), S. 26-33. https://doi.org/10.1515/abitech-2019-1005 Piwowar, Heather; Priem, Jason; Larivière, Vincent; Alperin, Juan Pablo; Matthias, Lisa; Norlander, Bree et al. (2018): The state of OA: a largescale analysis of the prevalence and impact of Open Access articles. In: Peerj 6, e4375. https://doi.org/10.7717/peerj.4375

PwC EU Services (2018): Cost of not having FAIR research data. Luxembourg. https://doi.org/10.2777/02999

Rat für Informationsinfrastrukturen (2016): Leistung aus Vielfalt. Empfehlungen zu Strukturen, Prozessen und Finanzierung des Forschungsdatenmanagements in Deutschland. Göttingen. http://www.rfii.de/?p=1998 
Revez, Jorge (2018): Opening the Heart of Science: A Review of the Changing Roles of Research Libraries. In: Publications 6 (1), S. 9. https://doi. org/10.3390/publications6010009

Schlosser, Melanie (2019): Academy-owned? Academic-led? Communityled? What's at stake in the words we use to describe new publishing paradigms (Library Publishing Coalition Blog). https://librarypublishing.org/alpd19-academy-owned-academic-led-community-led/, zuletzt aktualisiert am 06.02.2019.

Schmidt, Birgit; Bertino, Andrea; Beucke, Daniel; Brinken, Helene; Jahn, Najko; Matthias, Lisa et al. (2018): Open Science Support as a Portfolio of Services and Projects: From Awareness to Engagement. In: Publications 6 (2), S. 27. https://doi.org/10.3390/publications6020027

Schrenk, Philip; Mehlberg, Martin (2015): Die Bibliothek als Plattform für eine partizipative Informationskultur. Das Projekt „CoScience - Gemeinsam forschen und publizieren mit dem Netz" am Open Science Lab an der Technischen Informationsbibliothek (TIB). In: B.I.T. Online 18 (2), S. 105-115.

Senst, Henriette; Heldt, Katharina (2017): Die Rolle der Bibliothek im Forschungszyklus am Beispiel der Bibliothek des RKI. Ein Praxisbericht. In: GMS Medizin, Bibliothek, Information 17 (1-2). https://doi.org/10.3205/ mbi000384

Smith, Arfon M.; Katz, Daniel S.; Niemeyer, Kyle E. (2016): Software citation principles. In: PeerJ Computer Science 2 (2), e86. https://doi. org/10.7717/peerj-cs.86

Smith, MacKenzie; Barton, Mary; Branschofsky, Margret; McClellan, Greg; Walker, Julie Harford; Bass, Mick et al. (2003): DSpace. In: D-Lib Magazine 9 (1). https://doi.org/10.1045/january2003-smith

Strobel, Sven; Plank, Margret (2014): Semantische Suche nach wissenschaftlichen Videos - Automatische Verschlagwortung durch $\mathrm{Na}$ med Entity Recognition. In: ZFBB 61 (4-5), S. 254-258. https://doi. org/10.3196/18642950146145154

Stummeyer, Sabine (2018): OER - Open Educational Resources: Chancen für wissenschaftliche Bibliotheken durch den Einsatz von freien Lehr- und Lernmaterialien in der Hochschullehre. In: Frauke Schade und Ursula Georgy (Hg.): Praxishandbuch Informationsmarketing. Berlin, Boston: De Gruyter, S. 303-318. https://doi.org/10.1515/9783110539011020

Sugimoto, Cassidy R. (2019): New open-access journal Quantitative Science Studies (ISSI Blog). http://issi-society.org/blog/posts/2019/ january/the-international-society-for-scientometrics-and-informetrics- 
ends-support-for-journal-of-informetrics-launches-new-open-accessjournal-quantitative-science-studies/

Thiedig, Christoph (2018): Der Kerndatensatz Forschung: Aufbau, Anwendung und die Rolle der Bibliothek. In: GMS Medizin, Bibliothek, Information 18 (3). https://doi.org/10.3205/mbi000421

Tullney, Marco (2016): Wie messen wir unseren Open-Access-Anteil? In: „Der Schritt zurück als Schritt nach vorn - Macht der Siegeszug des Open Access Bibliotheken arbeitslos?" 7. Konferenz der Zentralbibliothek, Forschungszentrum Jülich, 14.-16. Juni 2016 : Proceedingsband. Zenodo. https://doi.org/10.5281/zenodo.888866

Tullney, Marco (2019): Erfolgreiches Journal Flipping: TIB unterstützt neue Zeitschrift Quantitative Science Studies. https://blogs.tib.eu/wp/ tib/2019/01/14/unterstuetzung-journal-flipping-qss/

Tulloch, Ayesha I. T.; Auerbach, Nancy; Avery-Gomm, Stephanie; Bayraktarov, Elisa; Butt, Nathalie; Dickman, Chris R. et al. (2018): A decision tree for assessing the risks and benefits of publishing biodiversity data. In: Nature ecology \& evolution 2 (8), S. 1209-1217. https://doi. org/10.1038/s41559-018-0608-1

Vicente-Saez, Ruben; Martinez-Fuentes, Clara (2018): Open Science now: A systematic literature review for an integrated definition. In: Journal of Business Research 88, S. 428-436. https://doi.org/10.1016/j. jbusres.2017.12.043

Villányi, Márton (2017): Ein freies Bibliothekssystem für wissenschaftliche Bibliotheken - Werkstattbericht der IST Austria Library. In: Informationspraxis 3 (1). https://doi.org/10.11588/ip.2017.1.35227

Weber, Karsten; Kleine, Nadine; Pallas, Frank; Ulbricht, Max-R. (2017): Technik zur Unterstützung von Citizen Science und Open Science. In: TATuP 26 (1-2), S. 25-30. https://doi.org/10.14512/tatup.26.1-2.25

Wilkinson, Mark D.; Dumontier, Michel; Aalbersberg, I. Jsbrand Jan; Appleton, Gabrielle; Axton, Myles; Baak, Arie et al. (2016): The FAIR Guiding Principles for scientific data management and stewardship. In: Scientific data 3, S. 160018. https://doi.org/10.1038/sdata.2016.18 Wilkinson, Mark D.; Sansone, Susanna-Assunta; Schultes, Erik; Doorn, Peter; Bonino da Silva Santos, Luiz Olavo; Dumontier, Michel (2018): A design framework and exemplar metrics for FAIRness. In: Scientific data 5, S. 180118. https://doi.org/10.1038/sdata.2018.118

Willinsky, John (2005): Open Journal Systems. In: Library Hi Tech 23 (4), S. 504-519. https://doi.org/10.1108/07378830510636300

Zellmann, Cedrik (2018): Bibliothekare und Informationswissenschaftler mit IT-Schwerpunkt in Deutschland - Bedarf, Aufgaben, Kompetenzan- 
forderungen und Vergleich der IT-Kompetenzen in Praxis und Studium. Hochschule Hannover. https://doi.org/10.25968/opus-1264

1 vgl auch The Open Access Citation Advantage Service (OACA): https:// sparceurope.org/what-we-do/open-access/sparc-europe-open-accessresources/open-access-citation-advantage-service-oaca/

2 vgl. auch Initiative for Open Citations: https://i4oc.org/

3 Zu dieser Entwicklung gehört beispielsweise der (langsame) Anstieg des Open-Access-Anteils an Publikationen, vgl. Piwowar et al. (2018).

4 Plan S: https://www.coalition-s.org/principles-and-implementation/

5 vgl. Wilkinson (2016)

6 vgl. auch Sugimoto (2019)

7 "assisting humans and machines in their discovery of, access to, integration and analysis of (...) scientific data (...)" (The FAIR Data Principles, https://www.force11.org/group/fairgroup/fairprinciples)

8 FAIR Data Publishing Group: https://www.force11.org/group/fairgroup

9 "They do, however, require clarity and transparency around the conditions governing access and reuse." (Mons 2017)

10 http://fairmetrics.org/

11 vgl. bspw. FOSTER Open Science, Open Metrics and Impact: https:// www. fosteropenscience.eu/foster-taxonomy/open-metrics-and-impact

12 vgl. auch Joint Roadmap for Open Science Tools: https://jrost.org/

13 Siehe etwa die SCOSS-Initiative (http://scoss.org/), die 2,5\%-Initiative (Lewis et al. 2018) oder auch Peter Krakers \#DontLeaveltToGoogle-Kampagne (https://science20.wordpress.com/2018/09/10/ dontleaveittogoogle/).

14 European Open Science Cloud (EOSC): https://ec.europa.eu/research/ openscience/index.cfm?pg=open-science-cloud

15 High Level Expert Group on the European Open Science Cloud: https:// ec.europa.eu/research/openscience/index.cfm?pg=open-sciencecloud-hleg

16 Rat für Informationsinfrastrukturen (RflI): http://www.rfii.de/de/start/ 